\title{
Educación de la sexualidad preescolar: Un enfoque alternativo y participativo
}

\section{Education of prescholastic sexuality: An alternative and participa- tive view}

\author{
Elizabeth San Pelayo Ferrer ${ }^{1}$ \\ Universidad Santo Tomás, Chile
}

(Recepción: Noviembre 2007 - Aceptación: Marzo 2008)

\begin{abstract}
Resumen
El presente trabajo tiene como objetivo describir las particularidades de la sexualidad en la etapa preescolar, reposicionando esta dimensión dentro de la configuración de la personalidad. Se exponen algunos principios humanistas que permiten entender la educación de la sexualidad desde una mirada participativa y crítica. Palabras claves: Educación, sexualidad, preescolar.
\end{abstract}

\begin{abstract}
The objective of this paper is describe the particularities of the sexuality in the prescholastic stage. Putting this dimension inside of personality configuration. Some humanists principles are exposed to understand the sexuality education from a critic and participative point of view.

Key words: Education, sexuality, prescholastic stage.
\end{abstract}

\section{Sexualidad. Conceptualización}

La sexualidad es parte indisoluble de la vida misma, de su creación, del devenir de hombres y mujeres como seres humanos sexuados. Fuente inagotable de comunicación, erotismo, ternura, trasciende el hecho reproductivo por lo que se construye, proyecta y expresa en todas las dimensiones: individuo, pareja, familia y sociedad. Enriquecedora de las relaciones interpersonales, permite el encuentro con el otro, el contacto, la intimidad y el establecimiento de vínculos emocionales y afectivos.

Sin lugar a dudas la sexualidad como una compleja manifestación vital, va más allá de lo biológico y no podemos reducirla al marco de las relaciones interpersonales y sociales. "Su esencia se completa en la espiritualidad, en lo subjetivo, que existe como realidad concreta de la personalidad única y creadora, capaz de transformar el entorno físico y cultural y de transformarse a sí misma en la praxis cotidiana". (Castellanos \& González, 2003, p. 8).

La sexualidad y la personalidad son dos categorías que se encuentran imbricadas en un estrecho y activo vínculo: la sexualidad siempre tiene un carácter personalizado y la personalidad es sexuada.

La personalidad como sistema regulador de la psiquis y con potencialidades para el crecimiento y desarrollo, organiza y dirige la espiritualidad de los seres humanos al integrar los procesos y fenómenos psíquicos, dentro de los cuales la sexualidad juega un importante papel; a su vez cada uno de ellos adquiere significación a través de sus mecanismos reguladores.

1 Correspondencia: Elizabeth San Pelayo Ferrer. Dirección Postal: Código Postal Comuna Talca 3460000. Dirección: 5 Norte N 4010. Condominio Las Rastras, Talca. Teléfono: 76157447. E-mail: esanpelayo@santotomas.cl 
La sexualidad se construye y expresa en la personalidad, se va desarrollando al unísono con esta, y todos los procesos afectivos, cognitivos, actitudes, concepciones, necesidades y modos de actuación se van integrando dinámicamente en su estructura y funcionamiento

“La sexualidad es comprendida como una dimensión de la personalidad, como parte indisoluble del ser humano o un elemento constitutivo de la propia naturaleza que funciona como una unidad armónica” (Aller, 1994, p.181). Esta concepción ha sido trabajada por diferentes autores contemporáneos, pero sus primeros pasos fueron aportados a finales del siglo XIX y principios del $\mathrm{XX}$ por Sigmund Freud, que estudia este fenómeno con una visión sistémica.

A pesar de revitalizar el papel de la sexualidad dentro del contexto de la personalidad, enfatiza en la influencia de los impulsos sexuales como fuerzas que se desencadenan de las profundidades del ser humano y dirigen toda la conducta del individuo en una lucha continua entre instintos y lo socialmente aceptado.

Numerosos científicos y estudiosos del tema en la actualidad, comparten el criterio de vincular la sexualidad y la personalidad, así encontramos:

Nadie desconoce hoy, que la sexualidad es parte de la personalidad. Decir sexualidad, no nos puede inducir solamente a pensar en genitales o coitos, ella es la profundidad y extensión de la persona con sus sentimientos, conductas, valores, conocimientos, que conllevan el ser varón o mujer (Arriagada, 1990, p. 14).

Master, Johnson y Kolodny (1987), al hablar de la sexualidad se refieren a una dimensión de la personalidad y no, exclusivamente, a la aptitud del individuo para generar una respuesta erótica.

Coincidimos con la propuesta teórica explicativa de las doctoras Alicia González y Beatriz Castellanos que desde posiciones de partida de la escuela histórica cultural explican la sexualidad como configuración psicológica en su compleja integración con la personalidad.

El hecho de asumir la sexualidad como configuración subjetiva de la personalidad, lleva a plantearse que en cada etapa del desarrollo personológico se van a establecer relaciones peculiares entre sexualidad y personalidad, esto fundamenta la afirmación de que no puede ser entendida la sexualidad en las diferentes etapas de la vida sin analizar el desarrollo de las características personológicas en esta etapa, de hecho, la sexualidad no tiene el mismo sentido para el sujeto a lo largo de todo su desarrollo como personalidad (González Rey, 1995).

Desde esta perspectiva se define la sexualidad como una configuración psicológica de la personalidad, que se conforma y desarrolla a partir de la interacción sistémica de premisas biológicas y sociales que en ella intervienen, expresando de forma personalizada las vivencias afectivas, las actitudes, los conocimientos y modos de actuación que el individuo interioriza activamente en el contexto socio-histórico en el que vive y se desarrolla.

La reproducción constituye un valor inherente de la sexualidad, pero no puede reducirse al marco reproductivo, ya que su función no se limita sólo a dejar descendencia, sino que se convierte en multiplicadora de la cultura que se transmite de generación en generación.

Reproducirse presupone la interrelación de factores psicosociales tales como afectos, actitudes y decisiones que se encuentran ligados a la visión que tiene cada persona acerca de la maternidad y la paternidad, lo cual trasciende el proceso de fecundación y parto, alcanzando su máxima expresión en la educación y cuidado de los hijos/as.

La sexualidad tiene como componentes fundamentales la identidad de género, el rol de género y la orientación sexo-erótica los cuales interactúan constantemente entre sí y con otros estados dinámicos relacionados con la vida del ser humano, matizando las funciones de la sexualidad (reproducción, placer erótico y comunicación), los cuales se expresan en las diferentes dimensiones en dependencia del sentido que cobran para el sujeto los mismos. 
Se torna necesario entonces definir estos componentes psicológicos:

Identidad de género: Es la convicción, autoconciencia y sentimiento de la propia individualidad como hombre, mujer o ambivalente. Es la manera de vivenciar el hecho de pertenecer a uno u otro sexo.

Rol de género: Es la expresión pública de la identidad de género asumida mediante el desempeño de diversos papeles (padre, madre, esposo, esposa, amigo, amiga, etc.), los cuales son prescriptos y proscriptos culturalmente a través de los modelos genéricos que cada sociedad dictamina según el sexo.

Orientación sexo-erótica: Dirección de las preferencias sexuales, eróticas y afectivas hacia el otro sexo, el mismo o ambos.

El ser humano es biológicamente sexuado, portador de un conjunto de características anatomofisiológicas que lo distinguen como hombre o mujer. Pero a medida que se desarrolla la personalidad los individuos van aprendiendo a ser psicológicamente sexuados, a construir su identidad de género a partir de la elaboración personal que realizan de los valores y atributos sexuales y la apropiación de modelos considerados por la sociedad como masculinos o femeninos, por lo que adquiere un carácter prescriptivo valorativo.

La forma de expresión que tiene la identidad de género ante los demás, es el rol de género, el cual se manifiesta en la conducta de los individuos según la manera particular de cada uno de expresar su masculinidad, feminidad o ambivalencia, teniendo en cuenta los modelos que la cultura dicta para hombres y mujeres.

Esta inflexibilidad y estereotipación de lo femenino y masculino conduce a que los seres humanos no se manifiesten en muchas ocasiones de manera propia y libre, limitando la expresión de sus verdaderos deseos, necesidades y motivaciones. Es de vital importancia brindar desde las primeras edades alternativas que le permitan a las personas independizarse de los esquemas rígidos y desarrollen una personalidad sana que sea flexible y auténtica, liberándose de las ataduras que establecen los prejuicios y estereotipos.

Esta concepción de la sexualidad permite concebir el proceso educativo a partir del sentido que adquiere para el educando la comunicación, la interactividad con el adulto y los otros niños y niñas, resaltando el papel del ser humano en la construcción de su propia sexualidad, teniendo en cuenta la unidad de lo afectivo y lo cognitivo en el proceso de educación de la sexualidad.

\section{Un acercamiento a la sexualidad del preescolar}

La sexualidad a lo largo de la historia ha estado acompañada de mitos y tabúes debido a su carácter íntimo y privado, lo cual ha provocado que sea percibida en muchas ocasiones como algo que debe dejarse a la espontaneidad y bajo ninguna circunstancia ser educada.

Sobre todo en la etapa infantil este criterio se fortalece, existiendo la creencia popular que los niños y las niñas no gozan de este privilegio, condenando así sus manifestaciones y neutralizando sus intereses en ese sentido.

Sin embargo Freud y sus seguidores legitimaron la existencia de la sexualidad infantil como parte del desarrollo humano al afirmar: "De la concepción popular del instinto sexual forma parte la creencia de que falta durante la niñez, no apareciendo hasta el período de la pubertad. Constituye esta creencia un error de consecuencias graves, pues a ella se debe principalmente nuestro actual desconocimiento de las circunstancias fundamentales de la vida sexual” (Freud, 1972, p. 797).

Esta aseveración provocó un profundo cambio. La consistencia conceptual de sus afirmaciones les devolvió a los niños/as su carácter de seres humanos sexuados, sacándolos de la categoría de ángeles donde los había encerrado la tradición religiosa y filosófica, colocándolos de esta forma en su verdadero lugar. 
Nos referiremos brevemente a sus concepciones por la trascendencia que tienen dentro de los estudios referentes al tema. Toda su teoría se basa en la obtención de placer a través de zonas especiales en el desarrollo psicosexual, boca, ano y genitales, recorriendo los diferentes centros para la obtención del placer, a lo largo de los primeros años.

ETAPA ORAL (nacimiento a 1 año)

El niño/a recibe gratificación a través de los labios, boca, y actividades como morder, chupar, tragar. El niño/a orientará sus cimientos hacia su capacidad para formar relaciones estables con otras personas.

ETAPA ANAL (1 a 3 años)

La gratificación la recibe mediante la ejercitación del control muscular.

ETAPA FÁLICA (3 a 6 años)

El área genital se convierte en una parte del cuerpo sensitiva e interesante para el niño/a. Reconocen las diferencias entre los sexos y hay curiosidad por éstas. Los niños/as se vuelven celosos de la relación especial entre los padres y al mismo tiempo el niño se interesa en la madre y la niña en el padre (complejo de Edipo y Electra). A medida que se resuelven los complejos, el niño/a incorpora algunas de las cualidades de su padre y madre a la personalidad y comienza a desarrollar roles tipificados a su sexo y edad.

ETAPA DE LATENCIA (6 a'12 años)

La mayor fuente de energía proviene de la interacción del niño/a con el mundo exterior. Adquisición de conocimientos y destrezas que lo capacitan a enfrentarse con su medio ambiente.

ETAPA GENITAL (12 años en adelante)

Los cambios hormonales en el cuerpo llevan a su madurez sexual. Se mueven fuera de la dependencia de los padres, hacia un mayor interés por las demás personas fuera del grupo familiar. Los órganos genitales son la mayor fuente de placer, por eso invierte esa energía en buscar amistades y prepararse para el matrimonio.

A pesar de que Freud biologiza un tanto las etapas y absolutizó el carácter instintivo de la sexualidad, es un mérito suyo el percatarse que el infante va necesitando en su contacto con la madre y el vínculo afectivo que se establece entre ambos, satisfacer otras necesidades de carácter psicosocial como es el caso de ser mimado, arrullado, etc., señalando de esta forma el papel que juegan los padres en el desarrollo de la sexualidad.

La personalidad como la forma más elevada de organización que integra procesos, propiedades y fenómenos psíquicos, en la etapa preescolar se encuentra en un período de génesis y construcción en el cual comienzan a estructurarse determinadas formaciones.

Vigotsky es uno de los autores que enfatiza la importancia de los períodos de desarrollo en la estructura de la personalidad. Como nuevas formaciones de la edad debemos comprender aquel nuevo tipo de estructura de la personalidad y su actividad, aquellos cambios psíquicos y sociales que aparecen por primera vez en la actual etapa y que, en lo más importante y esencial, determinan la conciencia del niño, de sus relaciones con el medio, su vida interna y externa, toda la marcha de su desarrollo en el período actual. (González Rey, 1995).

Este período de construcción de la personalidad en la etapa preescolar comprende dos facetas: una de ellas es la nueva forma de comprender el mundo y el lugar que ocupa el infante dentro de este, lo cual origina nuevas formas de comportamiento, y la otra se relaciona con el desarrollo de la voluntad y la estabilidad de la conducta y su independencia con respecto a circunstancias externas.

En estas edades se complejiza y varía la actividad de niños y niñas, se desarrollan los procesos cognoscitivos, aumentando la motivación para obtener constantemente nuevos conocimientos. Otra característica propia de la etapa es el aumento de interés por los patrones de conducta, así al escuchar 
un cuento tratan de determinar quién es el bueno y quién el malo, aprenden reglas relacionadas con los hábitos higiénicos y cuidados del cuerpo, pasando a un primer plano la asimilación de reglas que deben seguirse en las relaciones mutuas con sus coetáneos. Comienzan a experimentar sentimientos de orgullo no ya por el cumplimiento de acciones favorecidas por el adulto, sino como consecuencia de sus cualidades positivas.

En los preescolares el juego tiene gran importancia para el desarrollo de la personalidad, reproduciendo en sus actividades los papeles e interrelaciones de los adultos, además de no limitarse al marco estrecho de las relaciones familiares, aumentando el vínculo comunicativo con otras personas, en particular con los infantes de sus mismas edades.

En la infancia las manifestaciones de la sexualidad se diferencian de otras etapas, las cuales están en correspondencia con su desarrollo evolutivo, al constituir la sexualidad una manifestación de la personalidad en formación. Es por ello que se hace imprescindible para su estudio partir del conocimiento de las regularidades del desarrollo psíquico infantil en estas edades.

A partir del nacimiento y durante el primer año de vida, la influencia familiar y las relaciones que se establecen con el niño/a, van determinando la relación social entre lo femenino y masculino, mediante el establecimiento de pautas culturales que asignan diferentes funciones según el sexo. Así se va conformando un mundo azul y rosado que se expresa en nombres, juguetes y formas de vestir, asegurándoles un rol de género que tipificará su conducta como hombres y mujeres. En esta primera etapa de la vida comienzan a surgir y desarrollarse los niveles primarios de la autoconciencia, el yo, núcleo central a partir del cual se conformará la personalidad. Parte imprescindible del yo, es la auto- percepción y autovaloración que va teniendo de su propio cuerpo, percatándose que forma parte de él mismo.

Durante el segundo año, al hacerse más independientes de los adultos gracias a la marcha y las relaciones con el mundo que los rodea, comienzan a descubrir las relaciones con objetos y sus funciones, etc. En esa misma medida comienzan a reconocerse a sí mismos por su aspecto externo, siendo capaces de identificarse como niño o niña, es decir reconocen su identidad sexual, la cual constituye uno de los procesos más importantes del desarrollo de la personalidad.

El reconocerse a sí mismos como seres sexuados se logra totalmente hacia finales del tercer año de vida, que es cuando comienzan a sentir curiosidad hacia las diferentes partes del cuerpo y descubren que los genitales son fuente de sensaciones placenteras. En esta misma etapa comienzan a compararse con los adultos, a identificarse con los roles que estos realizan de acuerdo al sexo: "soy mamá, hago lo que hace mamá”.

A partir de los 4 ó 5 años van tomando conciencia de la permanencia de la identidad, consolidando este proceso a los 6 ó 7 años. Este proceso de la adquisición de la identidad y el rol de género está íntimamente relacionado con la edad, y más concretamente, con el nivel de desarrollo. A medida que los niños/as adquieren otras capacidades (lenguaje, concepto del tiempo) van siendo cada vez más capaces de discriminar las diferencias asignadas al rol, autoclasificarse, saberse niño/a y reconocerse como hombre o mujer independientemente de apariencias superficiales (vestuario, accesorios, largo del pelo, etc.). Además van logrando discriminar lo cambiable y cultural (rol) de lo estable y biológicamente condicionado (identidad sexual).

En estas edades la conducta exploratoria se extiende a la observación y tocamiento de los genitales de otros infantes, siendo la causa de mayor atracción por el otro sexo, la curiosidad sobre las diferencias anatómicas. Además en este período realizan preguntas relacionadas con el origen de las personas, a raíz de situaciones cercanas como son el embarazo de alguna persona cercana a él/ ella o el nacimiento de un hermanito/a, así como las referidas al modo que ocurre el nacimiento y el placer sexual, las cuales con el decurso del tiempo van ganando en complejidad.

Otra de las características que evidencian los niños/as en estas edades, es la asunción del rol según el sexo. Los niños están más rígidamente tipificados que las niñas, entre estas es más frecuente el interés por las actividades de los niños y por los juguetes considerados tradicionalmente como 
masculinos. Los varones también se muestran más autosatisfechos con el rol que la sociedad les ha asignado, esto demuestra que niños/as perciben desde muy pronto que los roles no son igualitarios.

En este proceso de formación y desarrollo de la identidad y del rol de género, la familia y las instituciones educativas adquieren un significado vital, pues un elemento distintivo en este período es precisamente el papel mediador del adulto, lo que no quiere decir que se pierda en etapas posteriores, pero en estos momentos es fundamental sobre todo si consideramos que la afectividad es determinante en la configuración de la sexualidad.

En esta etapa de la vida, los adultos constituyen modelos a seguir, por lo que en la medida que manifiesten una sexualidad plena, libre de prejuicios en todas las esferas de la vida, así será la representación que tendrán sus hijos(as) de esta importante dimensión.

Es por todo lo anteriormente expuesto que se ha demostrado, que a medida que la educación rompa con estereotipos sexistas y favorezca el desarrollo de habilidades y potencialidades igualitarias para ambos sexos, permitiendo la libre expresión de pensamientos y sentimientos, estaremos formando hombres y mujeres más sanos que vivan su sexualidad de manera responsable y feliz.

\section{Hacia una concepción de educación sexual}

La educación en el decursar de la historia ha sido definida de diferentes formas: Transmisión de conocimiento, desarrollo de habilidades, instrucción, doctrina para perfeccionar a los niños/niñas, acción que permite el mejoramiento de la inteligencia, etc. Todas estas concepciones hacen énfasis en el aspecto cognitivo del proceso educativo, perdiendo de vista la riqueza y dimensiones que abarca la acción de educar.

Desde nuestra concepción, educar es preparar al ser humano desde las primeras etapas, insertándolo en el momento histórico que le tocó vivir como ser social que se apropia de forma personalizada de la cultura que legaron las generaciones que le antecedieron, desarrollando sus potencialidades para transformar el entorno y autotransformarse teniendo en cuenta la correspondencia entre las necesidades individuales y las sociales.

Las investigadoras cubanas Alicia González y Beatriz Castellanos, aportan una visión renovada de la educación al ubicar en el centro del proceso al ser humano como personalidad protagónica, integral, única, activa y creadora, por cuanto:

- $\quad$ Construye y reconstruye nuevos saberes y experiencias, a partir de sus saberes y experiencias anteriores, de sus necesidades y potencialidades, de sus propios patrones motivacionales, cognitivos y conductuales.

- Descubre un sentido especial y propio, una singular significación afectiva, en las ideas y los hechos, en los conocimientos, valores y preceptos conductuales, al vincularlos con su vida, sus problemas, intereses y expectativas.

- Crea y recrea nuevas realidades respecto a sí mismo, a los demás y su contexto; se trasciende, avanza hacia la autonomía y la autorrealización, se enriquece y crece, abriéndose nuevos horizontes que le permiten mejorar la calidad de vida individual y social. (Castellanos \& González, 2003, p. 143).

Educar la sexualidad es una necesidad de los seres humanos, obviarla sería negar una importante esfera de la vida, que aunque no constituye el centro exclusivo del desarrollo de la personalidad, sí es una de las configuraciones que proporciona realización plena y placer.

La educación sexual debe estar dirigida a potenciar una sexualidad sana que promueva estilos de vida saludables. Según la OPS, salud sexual no es más que: "La integración del ser humano de lo somático, lo emocional, lo intelectual y lo social de la conducta sexual, para lograr un enriquecimiento 
positivo de la personalidad humana que facilite sus potencialidades de comunicación y de dar y recibir amor” (Bedevia, 2002, p. 8).

Esto sólo puede ser posible si desde la infancia se fomenta una sexualidad sana que incluya los siguientes argumentos:

Reconocimiento de la actitud corporal de todas las zonas del cuerpo, para sentir, recibir y dar placer.

La ausencia de temores, sentimientos de vergüenza, culpabilidad y creencias infundadas que inhiban o perturben las relaciones sexuales.

Partimos de la conceptualización de educación sexual, aportada por Mariela Castro Espín en la conferencia magistral presentada en la clausura del III Congreso Cubano de Educación, Orientación y Terapia Sexual.

"Proceso social e individual que posibilita a las personas, tanto del sexo femenino como del masculino, adquirir un sistema orientador para la toma de decisiones informadas, conscientes y responsables que determinan favorablemente sobre su salud sexual a lo largo de toda la vida. Se inicia desde el nacimiento, dentro del grupo familiar, y transcurre durante toda la vida en los sucesivos vínculos que establecen las personas al interactuar con diferentes grupos. Ocurre de manera espontánea como parte del proceso de socialización del ser humano y puede estructurarse de forma dirigida por las vías formales y no formales de la educación” (Castro, 2001, p. 3).

Todos educamos la sexualidad, unos de manera formal, es decir dirigiendo las acciones de aprendizaje de manera consciente e intencionada y otros de manera informal, transmitiendo a través de costumbres, actitudes, silencios y hábitos de la vida cotidiana los modelos y representaciones propios de la sexualidad.

En este proceso la familia constituye el primer agente de socialización y el grupo de referencia más estable en cuanto a la formación de valores, puntos de vista, concepciones y actitudes. Es de vital importancia que las relaciones que se establezcan entre sus miembros estén basadas en el amor, el respeto mutuo, la colaboración y el complemento de los roles, porque es precisamente allí donde los niñas/os se apropian de los modelos que sobre lo femenino/masculino se tienen, y adquieren una visión positiva o negativa de la sexualidad.

Las instituciones educativas constituyen pilares importantes en la educación de la sexualidad desde las primeras etapas de la vida, en la formación de valores y cualidades morales de las niñas/ os; tal es el caso de los Círculos Infantiles, los cuales tiene decisivas influencias, por lo que se hace necesario que la familia apoye el trabajo de estos centros de manera que las acciones de ambos como agentes de socialización se integren y complementen para lograr el éxito en el proceso educativo.

Sin lugar a dudas, la observación e imitación de la conducta de los adultos (hermanos/as, padres/ madres, educadores/as) como modelos reales son fundamentales en el proceso de aprendizaje de la identidad y el rol de género, al constituir influencias permanentes cargadas de afectividad, regulando su aprendizaje a través de castigos o refuerzos de las conductas sexuales. Sin embargo, a pesar de tener gran impacto entre los/as infantes, existen otros modelos que influyen en las conductas sexuales de los niños/as, así encontramos:

- Los modelos simbólicos (visuales o literarios) ofrecen conductas sexuales y sus consecuencias en una unidad de tiempo y frecuencia que no se da en la realidad, permitiendo el establecimiento de relaciones causa/efecto, de esta manera aprenden lo que deben hacer o evitar.

- Los modelos intermediarios se manifiestan a través de objetos, vestidos, pendientes, representando el mundo de los adultos y expresando mensajes relacionados con la sexualidad.

- Los modelos ejemplares transmiten valores que se incorporan durante la infancia y son impuestos por los medios de comunicación social. 
A lo largo de la historia, la educación de la sexualidad dominante ha estado encaminada a proporcionar a los educandos grandes volúmenes de datos e información, percibiendo al individuo como un ente pasivo que solo debe recepcionar todo aquello que el profesor/a desde su posición de poder y de saber transmite, desconociendo el arsenal de experiencias y saberes individuales. Reproductora de los valores más tradicionales, perpetúa en muchas ocasiones los estereotipos sexistas que divorcian a hombres y mujeres que deben luego "convivir en armonía”, desconocedoras de las fuerzas informales que ejercen su influencia durante toda la vida, limitando el proceso educativo a la escuela como principal agente socializador.

Esta concepción es limitada desde nuestro referente, puesto que centra la atención en momentos parciales haciendo énfasis en el aspecto cognitivo del proceso, además de convertirse en generadora de una serie de consecuencias negativas, como son:

- $\quad$ Transmisión de roles estereotipados.

- $\quad$ Represión de la curiosidad sexual y el placer.

- $\quad$ Permanencia de tabúes y mitos.

- Identificación de sexualidad con genitalidad.

- Dificultades en la planificación familiar, con riesgos de abortos inducidos, embarazos precoces, matrimonios prematuros, hijos e hijas no deseados

Este tipo de educación, a la vez que imposibilita el desarrollo pleno de hombres y mujeres, entorpece la libre expresión de su sexualidad, aun en sociedades como la nuestra que promueve la igualdad y equidad entre los sexos.

Actualmente, teniendo en cuenta las definiciones de educación sexual tradicional, se promueve una nueva visión que centra su mirada en el papel protagónico y activo del educando, que posibilita el desarrollo de sus potencialidades y posibilidades personales a través de un proceso flexible, participativo y reflexivo que integra y compromete no sólo al educando/a como personalidad activa y creadora, sino a todos los agentes socializadores relacionados con éste.

Coincidimos con los principios del enfoque humanista crítico de la sexualidad y su educación aportados por las doctoras Alicia González y Beatriz Castellanos.

- Se ubica al ser humano en el centro del proceso vital y educativo como artífice de la construcción libre de su personalidad y su sexualidad.

- Se deposita toda la confianza en su capacidad de autodeterminación y autorrealización.

- Se prepara para que las alternativas de vida sean libres, plenas y responsables, comprometidas con su realidad, con una profunda conciencia crítica y dialéctica de la trascendencia de sus actos.

- Se propicia la articulación dinámica de sus necesidades y expectativas con las de su contexto con el menor sacrificio de su individualidad (González \& Castellanos, (2001, p. 157).

El enfoque humanista crítico de la sexualidad constituye un marco de referencia para abordar la educación sexual en la etapa preescolar, la cual no estaba alejada de las tendencias que ha tenido el proceso educativo de la sexualidad en América Latina, teniendo presente que aquí los tabúes y prejuicios son mayores, porque a pesar que en los documentos oficiales se habla de una educación de la sexualidad desde edades tempranas, esto aún no se ha concretado en la práctica, pues continúa arraigada la concepción de equivalencia entre sexo y sexualidad. 
La sexualidad alternativa y participativa plantea como postulado esencial el respeto a la individualidad del ser humano y sus vivencias sin separarlo del contexto. Esta posición propicia el desarrollo de la libertad responsable, ofrece opciones de vida que le permiten al sujeto elegir su propio destino favoreciendo la participación activa de los educandos.

Desde esta perspectiva se hace necesario que se establezca el diálogo entre educadores e infantes y que las relaciones que se desarrollen entre ambos se sustenten en la igualdad, donde si bien niños y niñas, son los protagonistas del aprendizaje, el maestro(a) sea un facilitador, un guía que oriente las potencialidades de los(as) preescolares en pos del crecimiento y el autodesarrollo, convirtiéndolos con su labor, en personas activas y creadoras.

Partiendo de esta concepción y teniendo en cuenta los puntos de vista expuestos en relación a la educación sexual así como los principios de la educación de la sexualidad alternativa y participativa que proponen B. Castellanos y A. González, se exponen los siguientes principios de la educación de la sexualidad en la edad preescolar.

\section{Carácter socializador y personalizado:}

El ser humano es un ser social, la relación que se establece en esta etapa con la sociedad, se produce a partir de la apropiación que hacen los niños/as en su relación con los adultos, de la cultura que se ha ido acumulando, matizándola con sus vivencias personales, de ahí la trascendencia de la educación sexual en los marcos de la familia y el Círculo Infantil. Es precisamente a partir de la interacción, que los niños/as comienzan a configurar la identidad y el rol de género, se trata de convertir al individuo en un sujeto activo y protagónico, donde el niño y la niña construyan su identidad y rol a partir de modelos flexibles que potencien el crecimiento individual al facilitar que cobre un sentido personal, logrando un compromiso afectivo, cognitivo y conductual.

\section{Carácter Humanista:}

Partimos del principio que el niño/a constituye el centro del proceso educativo y para ello es de suma importancia respetar su individualidad, al propiciar el desarrollo de su independencia y creatividad, utilizar al máximo sus potencialidades para ampliar de esta forma la zona de desarrollo próximo. Esto sólo se hace posible utilizando métodos educativos no directivos que tributen a la formación y desarrollo de una personalidad sana.

\section{Carácter Desarrollador:}

La educación de la sexualidad es desarrolladora cuando tiene en cuenta y parte de la situación de desarrollo actual de los/as infantes, respetando las particularidades de la edad y las características individuales de los niños/as para lograr a través de las situaciones psicopedagógicas el tránsito al nivel superior y el logro de la autonomía.

4. Vinculación con la vida:

No se concibe un sistema educativo alejado del contexto en que se desarrolla el sujeto, desvinculado de su propia realidad y de los fenómenos que acontecen a su alrededor. Es por ello que la sexualidad en la etapa preescolar debe ser educada en la misma medida que las otras dimensiones de la vida, pues esta constituye una forma de crecimiento y realización personal.

5. Carácter sistémico y sistematizado:

Para que la sexualidad se desarrolle adecuadamente se torna necesaria la integración permanente y sistémica de todas las instituciones y organismos que influyen en este proceso para garantizar la permanencia de los valores que se pretenden formar. 


\section{Referencias}

Aller, L. (1994). Programas estatales de educación sexual en El Salvador. Memorias del VI Congreso Colombiano de Sexología y educación sexual. Santiago de Cali, Colombia: Grupo editorial.

Arriagada, L. (1990). Educación sexual y familiar. En González, A. \& Castellanos, B. (2003). Sexualidad y género. Alternativas para su educación ante los retos del siglo XXI. La Habana, Cuba: Editorial Científico-Técnica.

Bedevia, A. (2002). Derechos sexuales. Juventud Rebelde 2, 3, 8-12.

Castro, M. (2001). Conferencia magistral presentada en la clausura del III Congreso Cubano de educación, orientación y terapia sexual, III Taller Iberoamericano de Educación sexual y orientación para la vida. I Simposio Latinoamericano para el estudio de la impotencia. La Habana, Cuba: Editorial Revolucionaria.

Freud, S. (1972). Obras completas. Madrid, España: Editorial Biblioteca Nueva.

González, A. \& Castellanos, B. (2001). Sexualidad, Diversidad y Educación. Conferencia magistral ofrecida en el II Taller Iberoamericano Educación Sexual y Orientación para la vida. I Simposio Latinoamericano para el estudio de la impotencia. La Habana, Cuba: Editorial CientíficoTécnica.

González, A. y Castellanos, B. (2003). Sexualidad y género. Alternativas para su educación ante los retos del siglo XXI. La Habana, Cuba: Editorial Científico-Técnica.

González Rey, F. (1995). Comunicación, Personalidad y Desarrollo. La Habana, Cuba: Editorial Pueblo y Educación.

Masters, W., Johnson, V. y Kolodny, R. (1987). La sexualidad humana. En González, A. \& Castellanos, B. (2003). Sexualidad y género. Alternativas para su educación ante los retos del siglo XXI. La Habana, Cuba: Editorial Científico-Técnica.

Vigotsky, L. S. (1995). El problema de la periodización por edades del desarrollo infantil. En González Rey, F. Comunicación, personalidad y desarrollo. La Habana: Editorial Pueblo y Educación. 